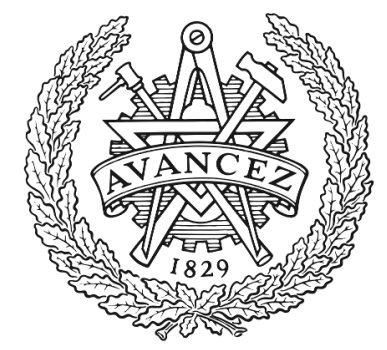

CHALMERS

UNIVERSITY OF TECHNOLOGY

\title{
Wide Bandwidth Terahertz Mixers Based On Graphene FETs
}

Downloaded from: https://research.chalmers.se, 2023-04-26 09:51 UTC

Citation for the original published paper (version of record):

Yang, X., Vorobiev, A., Jeppson, K. et al (2019). Wide Bandwidth Terahertz Mixers Based On Graphene FETs. International Conference on Infrared, Millimeter, and Terahertz Waves, IRMMW-THz, 2019-September. http://dx.doi.org/10.1109/IRMMW-THz.2019.8873869

N.B. When citing this work, cite the original published paper. 


\title{
Wide bandwidth terahertz mixers based on graphene FETs
}

\author{
Xinxin Yang ${ }^{1}$, Andrei Vorobiev ${ }^{1}$, Kjell Jeppson ${ }^{1}$, Jan Stake ${ }^{1}$, \\ Luca Banszerus ${ }^{2}$, Christoph Stampfer ${ }^{2}$, Martin Otto ${ }^{3}$ and Daniel Neumaier ${ }^{3}$ \\ ${ }^{1}$ Chalmers University of Technology, 41296 Gothenburg, Sweden \\ ${ }^{2} 2^{\text {nd }}$ Institute of Physics, RWTH Aachen University, 52074 Aachen, Germany \\ ${ }^{3}$ Advanced Microelectronic Center Aachen, AMO GmbH, 52074 Aachen, Germany
}

\begin{abstract}
In this work, we demonstrate wide RF and IF bandwidth resistive fundamental terahertz mixers based on graphene field-effect transistors. In the RF frequency range of 220-487 GHz, the 3-dB IF bandwidth is $32 \mathrm{GHz}$ and $56 \mathrm{GHz}$ for the mixers based on graphene field-effect transistors with the gate length of $1.2 \mu \mathrm{m}$ and $0.6 \mu \mathrm{m}$, respectively. The highest conversion efficiency is estimated to be $-28 \mathrm{~dB}$ at local oscillator power of 13 $\mathrm{dBm}$. This shows that resistive fundamental terahertz mixers based on graphene field-effect transistors are promising for ultrawide band-width applications.
\end{abstract}

\section{INTRODUCTION}

$\mathrm{W}$ ITH ever-increasing mobile internet traffic, terahertz $(\mathrm{THz})$ wireless communication technology is attracting great interest, since allowing for larger absolute bandwidth $[1,2]$. Being key components in any communication system, a THz mixer with wide RF and IF bandwidths is highly demanded. However, the circuit design and fabrication of the wide bandwidth $\mathrm{THz}$ mixers are challenging tasks. In recent years, it was shown that the graphene field-effect transistors (GFETs), owing to their linear output characteristics and relatively high carrier mobility, have a great potential for development of the GFET based high-frequency mixers [3-5]. But most of the demonstrated GFET mixers reveals rather narrow RF and/or IF bandwidths [6]. In this work, we experimentally demonstrate the GFET-based resistive fundamental $\mathrm{THz}$ mixers with wide instantaneous $\mathrm{RF}$ and $\mathrm{IF}$ bandwidths.

\section{RESULTS}

The THz mixers based on the GFETs were fabricated on Si substrate using the high-quality CVD graphene [7]. An optical micrograph of the mixer chip is shown in Fig. 1. Two versions of the mixers based on GFETs with different gate length $\left(L_{\mathrm{g}}\right)$ of 0.6 and $1.2 \mu \mathrm{m}$ and the same total gate width of $20 \mu \mathrm{m}$ (two 10

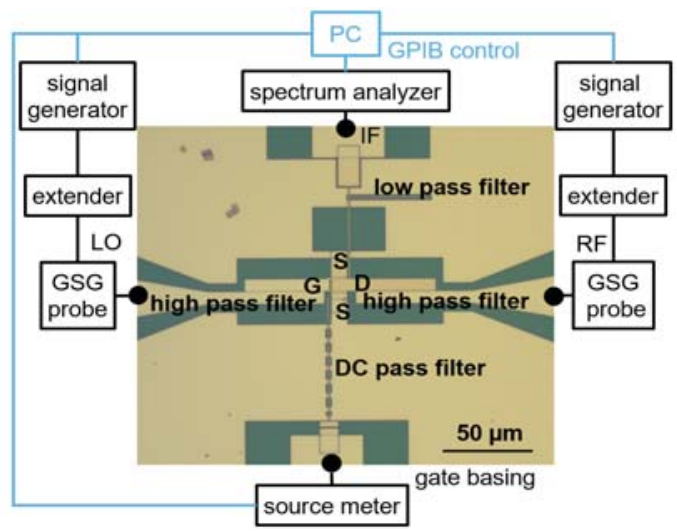

Fig. 1. Optical micrograph of a GFET mixer with $L_{\mathrm{g}}$ of $1.2 \mu \mathrm{m}$, together with a schematic block diagram of the on-wafer measurement setup. $\mu \mathrm{m}$ wide gate fingers in parallel) have been fabricated and analyzed. Coupled line high-pass filters are implemented at the LO and RF ports. The low-pass filter at the IF port consists of a quarter wavelength open stub and stepped-impedance lines. The DC pass-filter is implemented by a high-impedance transmission line. Full-wave EM simulations using CST microwave studio were applied for optimizing layout dimensions. The mixers were characterized on-wafer at room temperature. A schematic block diagram of the on-wafer measurement setup is shown in Fig. 1. The LO and RF signals obtained from Virginia Diodes vector network analyzer extenders were fed to the mixer by $\mathrm{T}$-Wave probes from FormFactor. The IF signals were measured using infinity probes from FormFactor and an FSU50 spectrum analyzer from Rohde \& Schwarz. Equipment was controlled remotely by computer via GPIB. The DC performance was measured using a Keithley 2604B Source meter. The S-parameters were measured between the LO and RF ports using a Keysight PNA-X N5247A with a thru-reflect-line (TRL) calibration procedure in the WR 2.2 and WR 3.4 frequency ranges. The TRL calibration kit was designed and fabricated on the same substrate as the GFET mixers.

Fig. 2 shows the measured and modeled drain-source resistance $\left(R_{\mathrm{ds}}\right)$ versus the gate voltage overdrive from the Dirac point $\left(V_{\mathrm{gs}}-V_{\mathrm{dir}}\right)$ of a GFET mixer with $L_{\mathrm{g}}$ of $1.2 \mu \mathrm{m}$. The $R_{\mathrm{ds}}$ is calculated using the transfer characteristic measured at a drain-source voltage $\left(V_{\mathrm{ds}}\right)$ of $0.05 \mathrm{~V}$. The electron/hole mobility $\left(\mu_{\mathrm{e} / \mathrm{h}}\right)$, the residual carrier concentration $\left(n_{0}\right)$, and the contact resistance $\left(R_{\mathrm{c}}\right)$ extracted via fitting the drain resistance model [8], are listed in Table 1.

The corresponding measured and simulated reflection coefficient, $\Gamma=\left(Z_{\text {in }}-Z_{\mathrm{c}}\right) /\left(Z_{\text {in }}+Z_{\mathrm{c}}\right)\left(Z_{\mathrm{c}}=50 \Omega\right)$, seen looking into the drain port is shown in Fig. 2. The figure shows that $\Gamma$ data simulated in ADS is in good agreement with the measured data. For a resistive mixer, the maximum conversion efficiency $(C E)$ can be determined from $C E=\left(\Gamma_{\max }-\Gamma_{\min }\right)^{2} / \pi^{2}$ [9]. To maximize $C E$, the difference between $\Gamma_{\max }$ and $\Gamma_{\min }$ should be maximized,

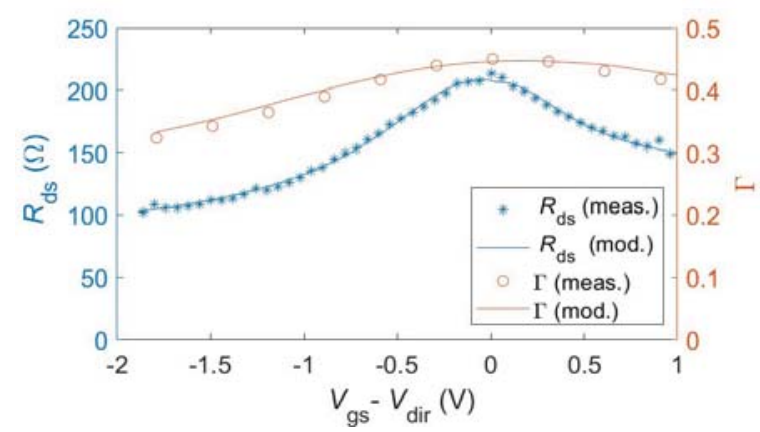

Fig. 2. Measured and modeled $R_{\mathrm{ds}}$ and $\Gamma$ curves versus $V_{\mathrm{gs}}-V_{\mathrm{dir}}$ at $V_{\mathrm{ds}}=0.05 \mathrm{~V}$ for a GFET mixer with $L_{\mathrm{g}}$ of $1.2 \mu \mathrm{m}$. 
Table 1 Extracted GFET DC model parameters

\begin{tabular}{|c|c|c|c|}
\hline & $\mu_{\mathrm{e} / \mathrm{h}}\left(\mathrm{cm}^{2} / \mathrm{Vs}\right)$ & $n_{0}\left(10^{12} \mathrm{~cm}^{-2}\right)$ & $R_{\mathrm{c}}(\Omega)$ \\
\hline$L_{\mathrm{g}}=0.6 \mu \mathrm{m}$ & $3200 / 3000$ & 1.5 & 70 \\
\hline$L_{\mathrm{g}}=1.2 \mu \mathrm{m}$ & $4300 / 4000$ & 1.3 & 120 \\
\hline
\end{tabular}

which corresponds to $R_{\mathrm{ds}, \min } \ll Z_{\mathrm{c}} \ll R_{\mathrm{ds} \text {,max }}$. Thus, GFETs with high $I_{\text {on }} / I_{\text {off }}$ ratios are required. In our case, with $\Gamma_{\max }=0.45$ and $\Gamma_{\min }=0.33$ obtained from the graph, this is far away from the ideal case with $\Gamma_{\min }=-1$ and $\Gamma_{\max }=1$. One reason is that $R_{\mathrm{ds}, \min }$ is larger than $Z_{\mathrm{c}}$ due to large contact resistances. In addition, effects of the GFET gate capacitance and parasitic circuit capacitances reduce the difference between $\Gamma_{\min }$ and $\Gamma_{\max }$ at high frequencies.

Fig. 3 shows the measured and simulated conversion efficiency versus the LO power $\left(P_{\mathrm{LO}}\right)$ for the GFET mixer with $L_{\mathrm{g}}$ of $0.6 \mu \mathrm{m}$. High LO power is required to properly pump the mixer between the on and the off states, and to maximize the $C E$. The measured and simulated $C E$ is up to $-50 \mathrm{~dB}$ at $P_{\mathrm{LO}}$ of $-11 \mathrm{dBm}$. The simulated $C E$ is slightly higher than the measured $C E$ probably due to a shift of the optimal biasing point. The highest $C E$ of the GFET mixers is simulated to be $-28 \mathrm{~dB}$ at $P_{\mathrm{LO}}$ of $13 \mathrm{dBm}$, which is already approaching to the best semiconductor analogues [10]. To compete with other technologies, further optimizations are required. As it can be seen from Fig. 3, the $C E$ can be as high as $-21 \mathrm{~dB}$ assuming 2 times lower contact resistance, which is possible to achieve using our recently developed technology [7].

Plots of the normalized conversion efficiency of the GFET mixers with $L_{\mathrm{g}}=0.6 \mu \mathrm{m}$ versus the IF frequency with LO frequency of $220 \mathrm{GHz}$ are shown in Fig. 4. The corresponding data for a GFET mixer with $L_{\mathrm{g}}=1.2 \mu \mathrm{m}$ is also shown in Fig. 4 for two $\mathrm{LO}$ frequencies, $220 \mathrm{GHz}$ and $438 \mathrm{GHz}$. It can be seen that the IF bandwidth is almost the same for the two LO frequencies owing to the wideband design of the mixer. The 3 -dB IF bandwidth $\left(f_{3 \mathrm{~dB}}\right)$, extracted by fitting the measured $C E$ with $C E=C E_{0}-20 \log \left(f_{\mathrm{IF}} / f_{3 \mathrm{~dB}}\right)^{2}$, was found to be around $56 \mathrm{GHz}$ and $35 \mathrm{GHz}$ for the GFETs with $L_{\mathrm{g}}$ of $0.6 \mu \mathrm{m}$ and $1.2 \mu \mathrm{m}$, respectively. These $f_{3 \mathrm{~dB}}$ values are comparable with those of the mixers made by semiconductor technologies, such as silicon CMOS mixers and Schottky-barrier diode THz mixers $[10,11]$.

\section{SUMMARY}

Using a wideband design, we have demonstrated that it is

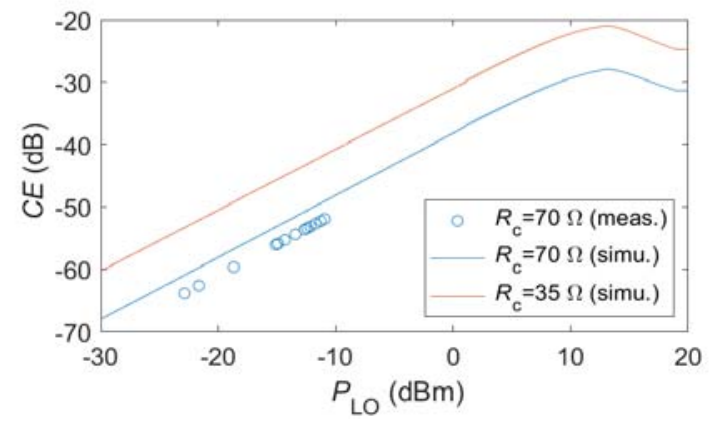

Fig. 3. Measured and simulated conversion efficiency versus LO power of the mixer with $L_{\mathrm{g}}$ of $0.6 \mu \mathrm{m}$ at $V_{\mathrm{gs}}-V_{\mathrm{dir}}=-0.5 \mathrm{~V}$, with an $\mathrm{LO}$ frequency of $220 \mathrm{GHz}$ and an RF frequency of $221 \mathrm{GHz}$. Also shown is a simulation of what the conversion efficiency would be with half the contact resistance (top curve). possible to develop GFET based THz mixers with wide RF and IF bandwidths, simultaneously. Therefore, we believe that this work will serve as platform for further development and corresponding improvements of GFET based mixers in terms of conversion efficiency, linearity and noise performance.

\section{ACKNOWLEDGMENT}

This work received funding in part from the European Union's Horizon 2020 research and innovation programme Graphene Flagship under grant agreement No 785219 (GrapheneCore2).

\section{REFERENCES}

[1]. S. Koenig, et al., "Wireless sub-THz communication system with high data rate," Nat. Photonics, vol.7, pp. 977-981, 2013. DOI: 10.1038/NPHOTON.2013.275

[2]. J. Federici, et al., "Review of terahertz and subterahertz wireless communications," J. Appl. Phys., vol. 107, pp. DOI:10.1063/1.3386413

[3]. A. A. Generalov, et al., "A heterodyne graphene FET detector at $400 \mathrm{GHz}$," in $42^{\text {nd }}$ International Conference on Infrared, Millimeter, and Terahertz Waves (IRMMW-THz), pp. 1-2, Cancun, Mexico, Aug.27-Sept. 1, 2017. DOI: 10.1109/IRMMW-THz.2017.8067234

[4]. M. Bonmann, et al., "An integrated 200-GHz graphene FET based receiver," in $43^{\text {rd }}$ International Conference on Infrared, Millimeter, and Terahertz Waves (IRMMW-THz), pp. 1-3, Nagoya, Japan, Sept. 9-14, 2018. DOI: 10.1109/IRMMW-THz.2018.8510069

[5]. O. Habibpour, et al., "A W-band MMIC resistive mixer based on epitaxial graphene FET," IEEE Microw. Wirel. Compon. Lett., vol. 27, pp. 168-170, 2017. DOI: 10.1109/LMWC.2016.2646998

[6]. M. A. Andersson, et al., "A 185-215-GHz subharmonic resistive graphene FET integrated mixer on silicon", IEEE Trans. Microw. Theory Techn., vol. 65, pp. 165-172, 2017. DOI:

10.1109/TMTT.2016.2615928

[7]. M. Bonmann, et al., "Graphene field-effect transistors with high extrinsic $f_{\mathrm{T}}$ and $f_{\max }$," IEEE Electron Device Lett., vol. 40, pp. 131-134, 2019. DOI: 10.1109/LED.2018.2884054

[8]. S. Kim et al., "Realization of a high mobility dual-gated graphene field-effect transistor with $\mathrm{Al}_{2} \mathrm{O}_{3}$ dielectric," Appl. Phys. Lett., vol. 94, pp. 062107, 2009. DOI:10.1063/1.3077021

[9]. K. Yhland, "Simplified analysis of resistive mixers," IEEE Microw. Wirel. Compon. Lett., vol. 17, pp. 604-606, 2007. DOI: 10.1109/LMWC.2007.901785 [10]. O. Inac, et al., "Double-balanced 130-180 GHz passive and balanced $145-165 \mathrm{GHz}$ active mixers in $45 \mathrm{~nm}$ CMOS," in 2011 IEEE Custom Integrated Circuits Conference (CICC), pp. 1-4, San Jose, CA, USA, Sept. 19-21, 2011. DOI: 10.1109/CICC.2011.6055323

[11]. J. Treuttel, et al., "330 GHz and $600 \mathrm{GHz}$ Schottky heterodyne systems for QPSK terahertz telecommunication," in 25th International Conference on Telecommunications (ICT), pp. 291-294, St. Malo, France, Jun. 26-28, 2018. DOI: 10.1109/ICT.2018.8464903

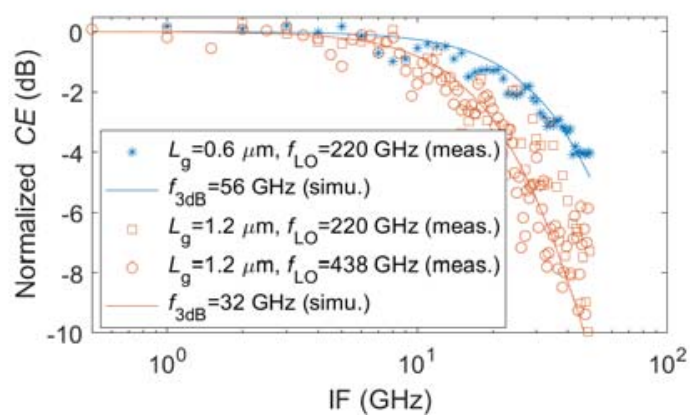

Fig. 4. Normalized conversion efficiency versus IF frequency. Symbols: measured data with zero gate bias and without drain bias. Lines: simulated data. For measurements obtained at an LO frequency of $220 \mathrm{GHz}$ the RF frequency was varied from $221 \mathrm{GHz}$ to $269 \mathrm{GHz}$, while for measurements obtained at an LO frequency of $438 \mathrm{GHz}$ the RF frequency was varied from $439 \mathrm{GHz}$ to $487 \mathrm{GHz}$. 